\title{
Brain imaging in dementia
}

\author{
Guendalina Bonifacio, ${ }^{1,2}$ Giovanna Zamboni ${ }^{1,2}$
}

${ }^{1}$ Nuffield Department of Clinical Neuroscience, University of Oxford, Oxford, UK

${ }^{2}$ Department of Biomedical, Metabolic, and Neural Sciences, University of Modena and Reggio Emilia, Italy

\section{Correspondence to} Dr Giovanna Zamboni, Nuffield Department of Clinical Neuroscience, FMRIB Centre, University of Oxford, John Radcliffe Hospital, Oxford OX3 9DU, UK;

giovanna.zamboni@ ndcn.ox.ac.uk

Received 5 October 2015 Revised 27 January 2016 Accepted 4 February 2016 Published Online First 1 March 2016
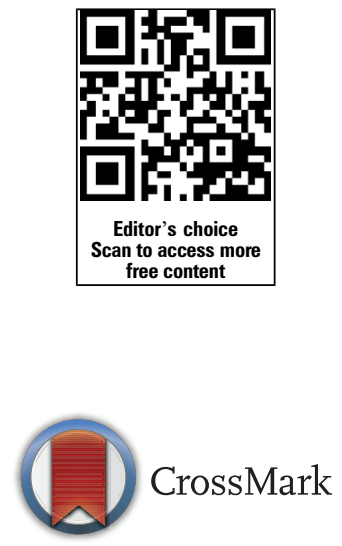

To cite: Bonifacio G, Zamboni G. Postgrad Med J 2016:92:333-340.

\section{ABSTRACT}

The introduction of MRI and positron emission tomography (PET) brain imaging has contributed significantly to the understanding of different dementia syndromes. Over the past 20 years these imaging techniques have been increasingly used for clinical characterisation and differential diagnosis, and to provide insight into the effects on functional capacity of the brain, patterns of spatial distribution of different dementia syndromes and their natural history and evolution over time. Brain imaging is also increasingly used in clinical trials, as part of inclusion criteria and/or as a surrogate outcome measure. Here we review all the relatively specific findings that can be identified with different MRI and PET techniques in each of the most frequent dementing disorders.

\section{INTRODUCTION}

The global prevalence of dementia in 2011 was estimated to be as high as 24 million, and it is expected to double every 20 years through to $2040 .{ }^{1}$ Dementia refers to a spectrum of diseases that affect the brain causing cognitive and behavioural disturbances. Cognitive problems include difficulties with memory, attention, language and other higher cortical functions. Behavioural changes include, as an example, apathy, aggressive behaviour, hallucinations and changes in social interaction. Because of these problems, patients' ability to relate with the world around them and to perform the acts of daily life is progressively affected. All dementia syndromes have relatively specific imaging findings that can be identified by one or more imaging techniques, thus contributing to the differential diagnosis.

Standard structural MRI allows identifying anatomical abnormalities of brain morphology for both grey and white matter and excluding other causes of cognitive decline such as the presence of tumour or hydrocephalus. It is acquired using highresolution T1-weighted, T2-weighted and diffusion tensor imaging (DTI) sequences. T1-weighted MRI is the preferred modality to examine focal loss of grey matter volume, usually indicated as atrophy, which is a common feature of neurodegenerative dementias (figure 1A, B). It has been shown that the spatial pattern of grey matter atrophy detected with T1-weighted MRI parallels the distribution of pathology specific for different dementia syndromes. As an example, in Alzheimer's disease (AD), atrophy parallels the distribution of neurofibrillary tangles in the brain. ${ }^{2} 3$ In addition, the degree and rate of volume loss are closely correlated with cognitive deficits. ${ }^{4}$ T2-weighted MRI sequences including fluid-attenuated inversion recovery are the preferred modality to examine white matter and periventricular lesions characterised by gliosis, ischaemia and oedema (figure 1C). Therefore, they are particularly useful in detecting vascular damage including features of small vessel disease, such as white matter hyperintensities (WMH) (also indicated as leukoaraiosis) and lacunar infarcts frequently found in vascular dementia ( $\mathrm{VaD}$, see the specific paragraph below), or other white matter abnormalities typical of rare, rapidly progressive dementia syndromes (progressive multifocal leukoencephalopathy and leukodystrophies, not discussed in this review). Paramagnetic-sensitive MRI sequences such as T2*-weighted gradient-recalled echo (GRE) or susceptibility-weighted sequences are most sensitive to detect microhaemorrhages (figure 1D), which are common in hypertension-associated $\mathrm{VaD}$ and in cerebral amyloid angiopathy (CAA) (not discussed in the present review). DTI measures the random motion of water molecules allowing inferences about the integrity of white matter tracts. It is currently used only in research settings to delineate the structural connectivity and axonal organisation of the brain.

Functional MRI (fMRI) provides information regarding the brain's functional status. With blood oxygen level-dependent (BOLD) fMRI, it is possible to design activation maps that show which parts of the brain are engaged when performing a task (task-related fMRI) or at rest (resting fMRI $(\mathrm{r}$-fMRI) $) .^{5}$ The $\mathrm{r}$-fMRI easy to be acquired even in patients with severe cognitive impairment, has been recently introduced to study large-scale networks that are coactivated and functionally connected, named resting state networks ${ }^{6} 7$ (figure 2). It has been shown that different networks may be specifically affected by different dementia syndromes, suggesting a potential role for this technique in the differential diagnosis of dementias. However, fMRI (either task-based or resting) is still only used in research settings to compare groups of patients relative to healthy controls, and cannot yet be used to get clinical information from single patients given the high variability of BOLD response between subjects, ${ }^{8}$ and therefore needs further validation before becoming clinically exploitable.

Other types of functional imaging are positron emission tomography (PET) and single photon emission CT (SPECT) techniques, which respectively allow to study metabolism and perfusion of the brain, at rest or during the execution of a task (activation-based PET or SPECT). They are based on the usage of radioactive ligands. They provide patient-specific information that can help with the differential diagnosis in clinical settings. In addition, in research settings they allow better understanding of the molecular and chemical pathophysiology of the disease. PET imaging is considered to have higher specificity and sensitivity 

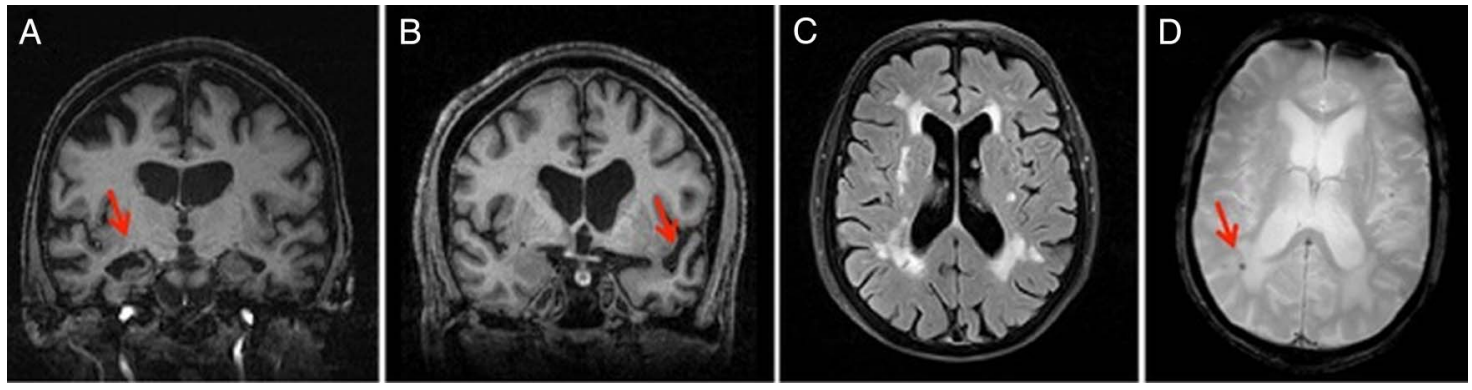

Figure 1 Structural MRI sequences. (A) T1-weighted MRI image of a patient with Alzheimer's disease, arrow pointing at right hippocampal atrophy; (B) T1-weighted MRI image of a patient with semantic dementia, arrow pointing at left temporal pole atrophy; (C) fluid-attenuated inversion recovery image of a patient with vascular cognitive impairment and features of small vessel disease consisting of periventricular white matter hyperintensities; (D) T2*-weighted gradient-recalled echo image of a patient with vascular cognitive impairment, arrow pointing at a microbleed, another feature of small vessel disease.

than SPECT in the diagnosis of different dementia syndromes. ${ }^{9}$ PET allows the quantification of functional markers of neurodegeneration (eg, cerebral glucose metabolism), specific neuropathological aggregates (amyloid and tau) and other potential factors involved in pathogenesis of different dementia disorders (eg, neuroinflammation or neurotransmitter systems).

\section{ALZHEIMER'S DISEASE}

$\mathrm{AD}$ is the most common age-related neurodegenerative disease. Its earliest symptoms usually include episodic memory loss, followed by deficits in language and executive functioning. AD worsens over time causing progressive impairment in everyday functioning usually accompanied, in the later stages, by behavioural disturbances. Recently revised diagnostic criteria require the presence of progressive cognitive dysfunction in one or more cognitive domains (reported and objectively measured on testing) and of functional impairment on the ability to perform daily activities. ${ }^{10}$ In addition, these criteria also consider, if available, the presence of abnormal imaging biomarkers (see below) that can enhance the likelihood that the clinical syndrome of dementia is specifically due to $A D$ pathophysiology.

The histopathological hallmarks of $\mathrm{AD}$ are amyloid plaques containing $\beta$-amyloid protein $(\mathrm{A} \beta)$ and neurofibrillary tangles. ${ }^{11}$ These abnormalities are primarily localised in the hippocampus, temporal cortex and nucleus basalis of Meynert. ${ }^{12}$ Macroscopically, there is atrophy, which can be easily detected with T1-weighted structural MRI. Despite the central role of A $\beta$ and its toxic effects in initiating the pathological cascade that results in $\mathrm{AD}$, the clinical and neuropathological progression of $\mathrm{AD}$ seems to correlate more with the number and spread of neurofibrillary tangles. ${ }^{11}$ Neurofibrillary tangles start to deposit in the entorhinal cortex then expand to the hippocampus and amygdala and finally to the association cortex. This sequence of progression is reflected on MRI, which shows that the areas most early affected by atrophy are the medial temporal lobes (figure $1 \mathrm{~A}),{ }^{13}{ }^{14}$ which are among the earliest sites of pathologic involvement, ${ }^{15}$ followed by atrophy in medial parietal regions (posterior cingulate and precuneus) and association areas of the frontal and lateral temporal lobes in the more advanced stages of the disease.

PET with ${ }^{18}$ fluorodeoxyglucose $\left({ }^{18} \mathrm{FDG}\right)$ in patients with AD shows a typical pattern of reduced cortical uptake in posterior and lateral temporal regions, medial parietal regions, particularly in the posterior cingulate gyrus and precuneus and medial temporal lobes including the hippocampus. ${ }^{16} 17$ With progression of the disease, metabolic deficit spreads to prefrontal association areas as well. ${ }^{18}{ }^{19}$ FDG hypometabolism parallels cognitive function and histopathological diagnosis of $\mathrm{AD}$ at autopsy, ${ }^{20} 21$ and can predict conversion to dementia in the elderly. ${ }^{22}$ Despite the fact that FDG-PET correlates well with the severity of the cognitive impairment, ${ }^{23}$ it is not specific for AD.

PET with tracers targeting insoluble fibrillar $A \beta$ (including the most studied Pittsburgh Compound-B (PiB), and more recently developed fluorine-18-labelled tracers (florbetaben, florbetapir and flutemetamol)) shows abnormal uptake in patients with AD. Areas found to show high uptake are the prefrontal cortex, precuneus, posterior cingulate cortex, lateral parietal and temporal cortex and striatum, with relatively lower tracer uptake in occipital cortex, globus pallidus and thalamus. ${ }^{24} \mathrm{~A}$ review by Johnson $e t a l^{8}$ has combined the data from five longitudinal studies $^{25-29}$ and has shown that $37 \%$ of people with some memory concern but no dementia (mild cognitive impairment (MCI)) will progress to $\mathrm{AD}$ within 3 years and increased uptake on amyloid imaging at baseline occurs in about 93\% of these patients with MCI. Indeed, it is thought that, by being a direct surrogate for $A \beta$ pathology, amyloid PET has the potential to detect the very beginnings of AD pathophysiology in the asymptomatic, predementia stage. However, whereas FDG-PET and structural MRI correlate significantly with the severity of cognitive decline, amyloid PET uptake does not increase with the progression of the disease, suggesting that amyloid deposition is an early event that triggers other pathological changes that are then responsible for further clinical progression. As patients develop $\mathrm{AD}$, the clinical and histological changes accelerate and proceed independently from amyloid accumulation. Postmortem studies have shown that neurofibrillary tangles and not $A \beta$ insoluble plaques more strongly correlate with neurocognitive impairment. For this reason, research teams in different countries are now focusing on the development of tau tracers for tau PET to aid with the diagnosis and treatment of AD. ${ }^{30}$ Tau is a constituent of microtubules and an alteration in tau phosphorylation leads to misfolding of microtubules with the formation of neurofibrillary tangles.

Activation-based PET and task-based fMRI detect changes in metabolism or blood flow while the subjects is engaged in a task, showing which parts of the brain are engaged when performing that specific task. As one of the earliest and most dominant symptoms of $\mathrm{AD}$ is the impairment of episodic memory, a number of functional imaging studies have used episodic memory tasks, demonstrating reduced activation of the medial temporal regions in patients with $\mathrm{AD}$ relative to controls. ${ }^{31} 32$ Increased prefrontal cortical activity in patients with $\mathrm{AD}$ relative to controls has also been reported, ${ }^{31}{ }^{32}$ but less consistently. More recently, resting fMRI has been used to study the integrity 


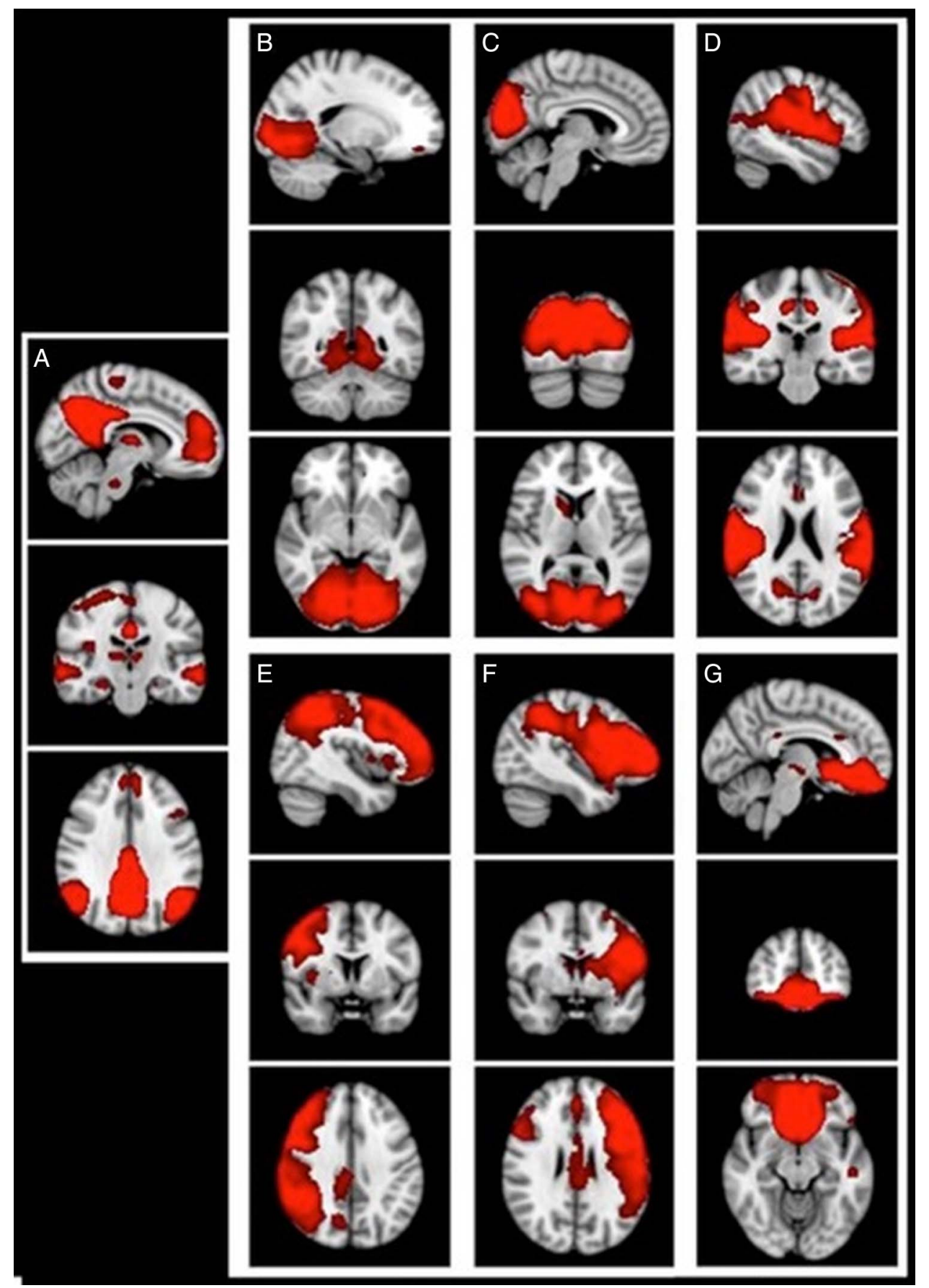

Figure 2 Spatial maps of resting state networks (RSN) obtained with resting functional MRI from our recent study. ${ }^{32}$ The three most informative orthogonal slices for each RSN are shown. (A) Default mode network; (B) ventromedial visual RSN; (C) dorsolateral visual RSN; (D) auditory RSN; (E) orbitofrontal RSN; (F) left frontoparietal RSN; (G) right frontoparietal RSN.

of resting state networks. Among them, the default mode network (DMN, figure 2A), which includes the posterior cingulate cortex, the hippocampus, the medial prefrontal cortex and the lateral parietal regions, has been shown to be specifically disrupted in patients with $\mathrm{AD}$ relative to controls. ${ }^{33}$ Other frontal and frontoparietal networks show increased functional connectivity in patients with $\mathrm{AD}$ relative to controls. ${ }^{34}$ However, task-based and resting functional imaging remain research techniques, as they cannot be used at the single-subject level and still need large validation studies before they can be translated and used in clinical practice.

\section{MILD COGNITIVE IMPAIRMENT}

MCI can be considered a stage in between normal ageing and dementia. ${ }^{35}$ Patients with MCI are more at risk of developing $\mathrm{AD}$ and other forms of dementia, thus this condition is also indicated as prodromal dementia. MCI is suspected when the patient, an informant or the clinician raise concern regarding the patient's cognitive performance, considered to be outside the normal range of function for the patient's age and educational background, but not yet impaired to be considered dementia. ${ }^{36}$ It has been calculated that the annual rate of conversion from $\mathrm{MCI}$ to $\mathrm{AD}$ ranges between $5 \%-10 \%{ }^{37}$ and 
$41 \%,{ }^{38}$ further confirming the gradual and progressive nature of $\mathrm{AD}$, where dementia represents the final stage of a series of pathological changes that have started decades before the onset of cognitive symptoms. In this process, it has been suggested that biochemical and imaging biomarkers become abnormal in a temporal order, reflecting the various stages of the neuropathological processes in the brain. ${ }^{39}$

Structural MRI in patients with MCI detects early focal atrophy relative to healthy controls, especially in the medial temporal lobe (MTL) (hippocampus and entorhinal cortex) and also in regions outside MTL, such as the lateral temporal lobes, the medial parietal lobes and lateral associative parietal areas. $^{40}{ }^{41}$ Several longitudinal studies have shown that structural MRI can differentiate patients with MCI who will progress to $\mathrm{AD}$ in the near future (converters) from patients with $\mathrm{MCI}$ who will remain stable (non-converters) and that the hippocampus has greater rates of change in converters than nonconverters, ${ }^{42}$ which increase close to the time of conversion. ${ }^{43}$

FDG-PET studies ${ }^{44}$ conducted on patients with MCI have shown reduced glucose metabolism first in memory-related areas like the MTL then in temporoparietal cortex, posterior cingulate and lateral parietal lobes.

Amyloid PET can also be a useful tool for early detection of the disease, since it has been proven that in the MCI phase amyloid accumulation has already reached the levels found in AD. ${ }^{44}$ Areas with high amyloid uptake are within the dorsolateral and medial frontal cortex, the temporal lobes and lateral and medial parietal regions. ${ }^{45}$ Higher levels of amyloid accumulation suggest a higher risk of conversion to AD. ${ }^{46}$ Some studies have shown that positivity to amyloid PET in patients with MCI is associated with a worst performance in memory tasks compared with amyloid-negative $\mathrm{MCI} .{ }^{47}$ However, there are conflicting studies on the role of amyloid deposition as a feature of progression to dementia ${ }^{42}$ or part of normal ageing. ${ }^{48}$ Approximately $10-30 \%$ of normal ageing patients have positive amyloid PET findings. ${ }^{43}$ Despite this, longitudinal studies have shown that patients with normal cognition at baseline and positive amyloid PET have higher risk to progress to $\mathrm{AD}$ or other dementia than subjects with negative amyloid PET. ${ }^{49}$

As for task-based fMRI, patients with MCI show increased activation of the medial temporal lobes during the execution of memory tasks. ${ }^{50}$ This paradoxically increased activation has been interpreted as reflecting compensation processes engaged during the MCI phase to counteract the structural damage, which dissipate in the $\mathrm{AD}$ phase when the neuronal loss becomes more widespread than it can accommodate. However, we recently showed that the MTL hyperactivation frequently observed in MCI is non-specific for successful memory performance, but it also occurs when MCI subjects fail a memory task, suggesting that it may therefore represent maladaptive or dysfunctional mechanisms of compensation. ${ }^{35}$

\section{VASCULAR DEMENTIA}

The clinical significance of $\mathrm{VaD}$ has been recently revived after several studies showed the increasing contribution of vascular risk factors to the development of dementia, especially in later life, and the high prevalence of vascular lesions in postmortem studies of patients with dementia. ${ }^{51} 52$ The diagnosis of VaD involves the presence of significant history of cerebrovascular pathology and/or cerebrovascular risk factors, assessed clinically or using neuroimaging techniques. Recently, the definition of $\mathrm{VaD}^{53}$ has evolved into the clinical concepts of vascular cognitive impairment (VCI) ${ }^{54}$ or vascular cognitive disorder, ${ }^{55}$ which refer to the whole spectrum of conditions in which there is both cognitive impairment and either clinical evidence of previous stroke or imaging evidence of vascular brain injury, either in isolation (pure vascular disease) or in association with other pathologies (mixed diseases).

T2-weighted structural MRI techniques play an important role in the detection of vascular abnormalities associated with VCI. Features of small vessel disease (the most frequent pathology found in $\mathrm{VaD}$ ) including leukoaraiosis or $\mathrm{WMH}$ (figure 1C), microhaemorrhages or microbleeds and lacunar infarcts ${ }^{56}$ have all been associated with risk of cognitive decline. ${ }^{57}$ WMH have been largely associated with cognitive, mood, gait and urinary problems in cross-sectional studies ${ }^{58}$ and with global functional decline in longitudinal studies. ${ }^{59}$ In addition, they have been associated with an increased risk of stroke, cognitive decline (especially in the executive function and processing speed domains) and death ${ }^{60}$ in the general population. However, it seems that they are not independently associated with cognitive decline in populations at risk of dementia (ie, from memory clinics) unless other structural lesions are also present. ${ }^{6162}$ The presence of lacunar infarcts on structural MRI is associated with immediate and prospective cognitive decline depending on the number of lacunes ${ }^{63}$ and their location. ${ }^{64}$ Silent lacunar infarct (incidentally found on MRI in absence of clinical history of cerebrovascular events) are detected in $20 \%$ of healthy individuals and associated with increased risk of subsequent stroke and dementia. ${ }^{65}$ Cerebral microhaemorrhages or microbleeds, which are small hypointense lesions visible on paramagnetic-sensitive MRI sequences (figure 1D), are also features of small vessel disease and are found in CAA, and also in cognitively healthy adults. Their number and lobar localisation have been associated with cognitive dysfunction mainly affecting executive functions. ${ }^{66}$ However, their prognostic value on longterm cognitive outcome remains controversial. ${ }^{67}$ Lastly, hippocampal atrophy detectable with T1-weighted structural MRI is also seen in patients with $\mathrm{VaD} / \mathrm{VCI}^{68}$ and longitudinal studies have argued that it is the best predictor of poststroke dementia. ${ }^{69}$

The predictive value of the above features of small vessel disease detectable with standard structural MRI is however limited by the lack of precise correlation with the degree of cognitive impairment, ${ }^{70}$ and by insufficient specificity for vascular pathology. ${ }^{71}$ DTI has been shown to better correlate with cognitive deficits (particularly executive functions), ${ }^{72}$ supporting the notion that cognitive dysfunction in the $\mathrm{VaD} / \mathrm{VCI}$ spectrum is due to the disruption of subcortical white matter pathways. ${ }^{73}$ However, it has mainly been used in research settings in comparisons between patients with $\mathrm{VaD} / \mathrm{VCI}$ and healthy controls.

In the context of VaD/VCI, amyloid PET, which has a high negative predictive value, is used to distinguish pure vascular from mixed (with both $\mathrm{AD}$ and vascular pathology) forms of dementia. Thus, the absence of amyloid PET uptake in patients with dementia suggests a pure vascular origin (ie, younger adults following stroke and with persistent cognitive impairment for more than 3 months ${ }^{74}$ ). However, the division between $\mathrm{AD}$ and $\mathrm{VaD}$ based on amyloid and cerebrovascular disease can be an extreme classification, since the two conditions have a common pathophysiology. Studies have shown that non-genetic $\mathrm{AD}$ is caused by vascular risk factors and therefore, a clear distinction is often not possible. ${ }^{75}$

The fMRI techniques including r-fMRI have not been extensively used in $\mathrm{VaD}$, even in research settings, mainly due to previous methodological limitations in accounting for the effect of concomitant structural vascular lesions (WMH, lacunar infarcts or larger strokes). A few recent studies, however, showed that 
higher volumes of WMH are associated with reduced functional connectivity and activation of frontal regions. ${ }^{76} 77$

\section{FRONTOTEMPORAL DEMENTIA}

Frontotemporal dementia (FTD) has a higher prevalence than AD among those aged below 60 years. $^{78}$ In 1998, Neary et al described clinical diagnostic criteria for FTD, ${ }^{79}$ distinguishing three different variants: behavioural FTD, semantic dementia (SD) and progressive non-fluent aphasia (PNFA).

The behavioural variant of FTD presents with behavioural changes including disinhibition, emotional blunting and detachment and alterations in social behaviour. On T1-weighted structural MRI, patients present focal atrophy in the frontal lobes, in particular in the medial, dorsolateral and orbitofrontal regions, compared with both patients with $\mathrm{AD}$ and age-matched controls. $^{80} 81$

With FDG-PET it is possible to differentiate FTD from AD. In $\mathrm{AD}$, FDG-PET shows hypometabolism mainly in the parietal regions bilaterally, whereas in FTD it shows reduced metabolism in the frontal and temporal regions.

Recent r-fMRI studies have shown that patients with behavioural variant of FTD have decreased functional connectivity in the salience network, which is thought to be important in social cognition and includes the anterior cingulate and fronto-insular regions $^{82}$ and increased functional connectivity in the DMN, with a pattern opposite to the one found in patients with AD.

SD and PNFA are aphasic variants of FTD and present mainly with language disturbances. SD is characterised by fluent language and severe semantic memory impairment. PNFA by non-fluent language, agrammatism and apraxia of speech. On T1-weighted structural MRI, SD is associated with unilateral and asymmetrical (either left or right) temporal lobe atrophy, particularly affecting anterior temporal regions and the temporal pole, with relative preservation of frontal lobe volumes (figure 1B). PNFA is instead associated with focal atrophy of the left posterior and inferior frontal regions and left perisylvian regions. $^{83}$

FDG-PET in SD demonstrates hypometabolism of the temporal lobes, particularly in the anterior and lateral temporal regions, and also in the hippocampus and amygdala. ${ }^{84}$ PNFA presents with a similar pattern, except that the left side is the most affected, and the hypometabolism involves more posterosuperior regions of the temporal lobe, and the posterior and inferior regions of the left frontal lobe.

The r-fMRI studies have shown that SD targets the left temporal polar network, while the PNFA involves mainly the left frontoparietal network. ${ }^{83}$ The former type of dementia presents, therefore, with a reduced connectivity between the frontal and temporal/limbic areas, but with an increased connectivity in the prefrontal cortex. These findings are supported by $\mathrm{fMRI}$ studies on patients with SD, which have demonstrated an altered activation during tasks such as sound, autobiographical memory and language tasks. Patients with PNFA, on the other hand, have reduced activation, on fMRI, during sentence reading and comprehension. ${ }^{84}$

\section{DEMENTIA WITH LEWY BODIES}

Dementia with Lewy bodies (DLB) is defined as a synucleinopathy, since it is formed by insoluble $\alpha$-synuclein, which aggregates to form the Lewy bodies (LBs) - the major pathological feature of DLB. LBs are round eosinophilic inclusions seen microscopically in cortical and subcortical regions of patients with DLB and their density correlates with the severity of the disease. ${ }^{85}$ Clinically, DLB presents with dementia, parkinsonism and visual hallucinations. ${ }^{86}$ Other suggestive clinical features comprehend rapid eye movement sleep behaviour disorders, unexplained falls and severe neuroleptic sensitivity. ${ }^{86}$

On T1-weighted structural MRI, patients with DLB show less typical focal atrophy of the hippocampus and other medial temporal lobe structures relative to patients with AD. ${ }^{87}$ Structural MRI studies on patients with DLB have shown a more extensive atrophy in cortical and subcortical structures such as striatum, substantia innominata, hypothalamus and dorsal midbrain ${ }^{88}$ relative to $\mathrm{AD}$ or healthy controls. However, at the singlesubject level, focal atrophy and other structural changes, such as WMH, remain not helpful in the differential diagnosis of DLB with other types of dementia.

DTI studies showed increased damage in the corpus callosum and pericallosal areas ${ }^{89}$ in patients with DLB compared with controls. In the same way as T1-weighted MRI, the utility of this technique in supporting a diagnosis of DLB at the singlesubject level still needs to be validated.

PET and SPECT imaging can be helpful to target dopamine receptors and detect dopamine transporter loss (DaTscan) in the basal ganglia. If abnormal, it suggests diagnosis of parkinsonism, allowing to distinguish, as an example, DLB from AD. Decreased dopamine transport in the caudate is associated with cognitive symptoms, whereas a reduction in the putamen is associated with motor symptoms. However, abnormal dopamine transporter SPECT and PET findings do not allow to distinguish DLB from other parkinsonism such as idiopathic Parkinson's disease. Altered dopamine uptake, together with the lack of atrophy in the MTL, represents two main features of DLB neuroimaging. ${ }^{90}$

FDG-PET in patients with DLB show significant hypometabolism in parietal and occipital areas, with relatively preserved metabolism in the temporal lobes, and with a pattern that is more 'posterior' and different from the one typical of $\mathrm{AD}$. These features indicate that occipital hypoperfusion is more specific for DLB. ${ }^{91}$

DLB is accompanied also by amyloid deposition similarly to AD. Amyloid PET uptake in patients with DLB is lower than the one observed in patients with $\mathrm{AD}$, but with a similar anatomical distribution to $\mathrm{AD}$ and there is a higher intersubject variability. ${ }^{92}$ However, compared with Parkinson's disease, DLB shows higher levels of amyloid deposition. ${ }^{93}$ Moreover, higher levels of amyloid in patients with DLB are associated with worse cognitive performance.

The r-fMRI studies demonstrated increased functional connectivity between the right posterior cingulate and other regions of the brain and reduced corticocortical connectivity. ${ }^{94}$ Finally, in adherence with occipital hypoperfusion and atrophy neuroimaging findings, fMRI studies highlighted reduced activation in the occipitotemporal lobe during visual tasks such as visual motion and face matching. ${ }^{94}$

\section{CONCLUSION}

We showed that different MRI and PET imaging techniques can detect relatively specific abnormalities in each of the most frequent dementia syndromes, and that the recent advent of new neuroimaging techniques such as resting fMRI, DTI and amyloid PET has allowed a rapid growth of research into dementia. However, imaging retains a supportive role in addition to the clinical assessment, and cannot be used in isolation to reach a diagnosis in people with dementia.

Obvious strengths of MRI are its availability in most Western countries, relatively low cost and non-invasiveness. ${ }^{95}$ On T1-weighted structural MRI, it is very useful to detect focal atrophy to help differential diagnosis of the different forms 
of neurodegenerative dementia. ${ }^{96}$ The significant correlation of atrophy with cognitive decline is indisputable.

Therefore, studies are trying to standardise and validate structural imaging and in particular hippocampal volumetry so that it can be adopted for diagnosis and in clinical trials. ${ }^{97}$ On the other side, structural MRI limitations comprehend the lack of specificity for underlying pathology of dementing syndromes, as atrophy patterns may overlap across different diseases. Structural MRI, moreover, takes long time to change and does not provide information about function like fMRI, therefore, limiting the possibility to detect dynamic, short-term changes in response to potential treatments. Further studies with advanced and novel MRI techniques will likely provide more information about pathology associated with the various dementia diseases.

Strengths of PET are that it targets molecules/receptors (ie, altered glucose metabolism and amyloid imaging) relevant to the pathogenesis of different dementia disorders, allowing preclinical diagnosis in presymptomatic individuals and improving differentiation from other types of dementia. In research settings, it can reveal important aspects of pathogenesis across the different dementia disorders, with the potential for novel therapeutic targeting.

Cost-effectiveness studies ${ }^{98} 99$ have demonstrated that neuroimaging techniques improve diagnosis and quality-adjusted life years. Evaluation of patients with MRI and/or PET scans can aid in the diagnosis without additional overall costs, allowing to increase diagnostic accuracy and to improve quality of life with the correct management and treatments.

It is also important to mention that if on one side MRI and PET scans are more widely available and more cost-effective, often patients cannot tolerate them due to claustrophobia, discomfort or behavioural problems. However, studies have shown that if subjects are well prepared prior the scanning through education and training, they are able to tolerate even long MRI sessions. $^{100}$

\section{Main messages}

- Neuroimaging techniques are useful in the diagnosis and differential diagnosis of different types of dementia and facilitate the understanding of pathological processes underlying different dementias.

- The main neuroimaging techniques currently used in clinical settings are structural MRI (to identify atrophy or white matter lesions) and positron emission tomography (PET) and single photon emission CT (to study altered metabolism and hypoperfusion).

- In typical Alzheimer's disease (AD), the first area showing atrophy or loss of volume is the medial temporal lobe, followed by medial parietal regions and association areas of the frontal and lateral temporal lobes.

- Amyloid PET uptake is an early biomarker of $A D$, but does not increase with the progression of the severity of dementia.

- Neuroimaging abnormalities in vascular dementia are diverse but non-specific. White matter hyperintensities correlate with cognitive impairment, but are also present in normal ageing patients.

- Frontotemporal dementia and its three main different subtypes have specific MRI and PET findings with focal atrophy and hypometabolism localised in frontal and temporal regions.

\section{Current research questions}

- What can emerging neuroimaging techniques reveal about the functional and molecular effects of different types of dementia?

- How can functional MRI improve the differentia diagnosis of dementia in the single patient?

- What are the potentials of resting functional MRI?

\section{Key references}

- Fox MD, Raichle ME. Spontaneous fluctuations in brain activity observed with functional magnetic resonance imaging. Nat Rev Neurosci 2007;8:700-11.

- McKhann GM, Knopman DS, Chertkow H, et al. The diagnosis of dementia due to Alzheimer's disease: recommendations from The National Institute on AgingAlzheimer's Association workgroups on diagnostic guidelines for Alzheimer's disease. Alzheimers Dement 2011;7:263-9.

- Zamboni G, Wilcock GK, Douaud G, et al. Resting functional connectivity reveals residual functional activity in Alzheimer's disease. Biol Psychiatry 2013;74:375-83.

- Albert MS, DeKosky ST, Dickson D, et al. The diagnosis of mild cognitive impairment due to Alzheimer's disease: Recommendations from The National Institute on AgingAlzheimer's Association workgroups on diagnostic guidelines for Alzheimer's disease. Alzheimers Dement 2011;7:270-9.

- Seeley WW, Crawford RK, Zhou J, et al. Neurodegenerative diseases target large-scale human brain networks. Neuron 2009;62:42-52.

\section{Self assessment questions}

Please answer True or False to the below statements.

1. Medial temporal lobe (MTL) atrophy is a hallmark biomarker of Alzheimer's disease (AD) and it can be detected with fluorodeoxyglucose positron emission tomography (FDG-PET).

2. In patients with mild cognitive impairment (MCI), structural MRI can detect focal atrophy even in areas outside the MTL.

3. Functional MRI is already currently used in a clinical setting for the differential diagnosis between the different types of dementia.

4. Leukoaraiosis or white matter hyperintensities (WMH) and microbleeds are MRI biomarkers highly specific for vascular dementia.

5. Amyloid deposition is a prerequisite of $A D$ and it can also be found in other types of dementia.

It is expected that in future different neuroimaging techniques will increasingly be combined in the management of people with or at risk of dementia with the aim of identifying the presence of a molecular abnormality, studying its effect on the brain structure and function, and measuring the effects of new treatments. Studies with more advanced MRI technologies and new 
PET tracers such as tau will help enable a better understanding of the pathology of neurodegenerative diseases and may be applied in clinical trials for the development of diseasemodifying drugs.

Contributors Both authors critically reviewed the existing literature, wrote the manuscript and agreed on its final version.

Competing interests None declared.

Provenance and peer review Commissioned; externally peer reviewed.

\section{REFERENCES}

1 Reitz C, Brayne C, Mayeux R. Epidemiology of Alzheimer disease. Nat Rev Neurol 2011;7:137-52.

2 Whitwell JL, Josephs KA, Murray ME, et al. MRI correlates of neurofibrillary tangle pathology at autopsy: a voxel-based morphometry study. Neurology 2008;71:743-9.

3 Vemuri P, Whitwell JL, Kantarci K, et al. Antemortem MRI based STructural Abnormality iNDex (STAND)-scores correlate with postmortem Braak neurofibrillary tangle stage. Neuroimage 2008;42:559-67.

4 Ridha BH, Anderson VM, Barnes J, et al. Volumetric MRI and cognitive measures in Alzheimer disease: comparison of markers of progression. J Neurol 2008:255:567-74.

5 Buckner RL, Andrews-Hanna JR, Schacter DL. The brain's default mode network: anatomy, function, and relevance to disease. Ann N Y Acad Sci 2008;1124:1-38.

6 Gusnard DA, Raichle ME, Raichle ME. Searching for a baseline: functional imaging and the resting human brain. Nat Rev Neurosci 2001;2:685-94.

7 Fox MD, Raichle ME. Spontaneous fluctuations in brain activity observed with functional magnetic resonance imaging. Nat Rev Neurosci 2007;8:700-11.

8 Johnson KA, Fox NC, Sperling RA, et al. Brain imaging in Alzheimer disease. Cold Spring Harb Perspect Med 2012;2:a006213.

9 Davison CM, O'Brien JT. A comparison of FDG-PET and blood flow SPECT in the diagnosis of neurodegenerative dementias: a systematic review. Int I Geriatr Psychiatry 2014;29:551-61.

10 McKhann GM, Knopman DS, Chertkow H, et al. The diagnosis of dementia due to Alzheimer's disease: recommendations from The National Institute on Aging-Alzheimer's Association workgroups on diagnostic guidelines for Alzheimer's disease. Alzheimers Dement 2011;7:263-9.

11 Goedert M, Klug A, Crowther RA. Tau protein, the paired helical filament and Alzheimer's disease. J Alzheimers Dis 2006;9(3 Suppl):195-207.

12 Teipel SJ, Flatz WH, Heinsen $\mathrm{H}$, et al. Measurement of basal forebrain atrophy in Alzheimer's disease using MRI. Brain 2005;128:2626-44.

13 Ashburner J, Friston KJ. Voxel-based morphometry-the methods. Neuroimage 2000;11(Pt 1):805-21.

14 Busatto GF, Diniz BS, Zanetti MV. Voxel-based morphometry in Alzheimer's disease. Expert Rev Neurother 2008:8:1691-702.

15 Braak H, Braak E, Bohl J, et al. Evolution of Alzheimer's disease related cortical lesions. J Neural Transm Supp/ 1998;54:97-106.

16 de Leon MJ, Convit A, Wolf OT, et al. Prediction of cognitive decline in normal elderly subjects with 2-[18F]fluoro-2-deoxy-D-glucose/positron-emission tomography (FDG/PET). Proc Natl Acad Sci USA 2001;98:10966-71.

17 Gibson GE. Interactions of oxidative stress with cellular calcium dynamics and glucose metabolism in Alzheimer's disease. Free Radical Biol Med 2002:32:1061-70.

18 Foster NL, Chase TN, Fedio P, et al. Alzheimer's disease: focal cortical changes shown by positron emission tomography. Neurology 1983;33:961-5.

19 Chase TN, Foster NL, Fedio P, et al. Regional cortical dysfunction in Alzheimer's disease as determined by positron emission tomography. Ann Neurol 1984;15 (Suppl):S170-4.

20 Furst AJ, Rabinovici GD, Rostomian AH, et al. Cognition, glucose metabolism and amyloid burden in Alzheimer's disease. Neurobiol Aging 2012;33:215-25.

21 Hoffman JM, Welsh-Bohmer KA, Hanson M, et al. FDG PET imaging in patients with pathologically verified dementia. J Nucl Med 2000;41:1920-8.

22 Mosconi L, Pupi A, De Leon MJ. Brain glucose hypometabolism and oxidative stress in preclinical Alzheimer's disease. Ann N Y Acad Sci 2008;1147:180-95.

23 Herholz K, Salmon E, Perani D, et al. Discrimination between Alzheimer dementia and controls by automated analysis of multicenter FDG PET. Neuroimage 2002;17:302-16.

24 Klunk WE, Engler H, Nordberg A, et al. Imaging brain amyloid in Alzheimer's disease with Pittsburgh Compound-B. Ann Neurol 2004;55:306-19.

25 Forsberg $\mathrm{A}$, Engler $\mathrm{H}$, Almkvist $\mathrm{O}$, et al. PET imaging of amyloid deposition in patients with mild cognitive impairment. Neurobiol Aging 2008;29:

$1456-65$.

26 Koivunen J, Pirttilä T, Kemppainen N, et al. PET amyloid ligand [11C]PIB uptake and cerebrospinal fluid beta-amyloid in mild cognitive impairment. Dement Geriatr Cogn Disord 2008;26:378-83.
27 Okello A, Koivunen J, Edison $\mathrm{P}$, et al. Conversion of amyloid positive and negative MCl to AD over 3 years: an 11C-PIB PET study. Neurology 2009;73:754-60.

28 Wolk DA, Price JC, Saxton JA, et al. Amyloid imaging in mild cognitive impairment subtypes. Ann Neurol 2009:65:557-68.

29 Jagust WJ, Bandy D, Chen K, et al. The Alzheimer's Disease Neuroimaging Initiative positron emission tomography core. Alzheimers Dement 2010;6:221-9.

30 Villemagne VL, Okamura N. Tau imaging in the study of ageing, Azheimer's disease and other neurodegenerative conditions. Curr Opin Neurobiol 2016;36:43-51.

31 Rombouts SA, Barkhof F, Veltman DJ, et al. Functional MR imaging in Alzheimer's disease during memory encoding. AJNR Am J Neuroradiol 2000;21:1869-75.

32 Johnson NA, Jahng GH, Weiner MW, et al. Pattern of cerebral hypoperfusion in Alzheimer disease and mild cognitive impairment measured with arterial spin-labeling MR imaging: initial experience. Radiology 2005;234:851-9.

33 Buckner RL, Snyder AZ, Shannon BJ, et al. Molecular, structural, and functional characterization of Alzheimer's disease: evidence for a relationship between default activity, amyloid, and memory. J Neurosci 2005;25:7709-17.

34 Zamboni G, Wilcock GK, Douaud G, et al. Resting functional connectivity reveals residual functional activity in Alzheimer's disease. Biol Psychiatry 2013;74:375-83.

35 Petersen RC, Smith GE, Waring SC, et al. Mild cognitive impairment: clinical characterization and outcome. Arch Neurol 1999:56:303-8.

36 Albert MS, DeKosky ST, Dickson D, et al. The diagnosis of mild cognitive impairment due to Alzheimer's disease: Recommendations from The National Institute on Aging-Alzheimer's Association workgroups on diagnostic guidelines for Alzheimer's disease. Alzheimers Dement 2011;7:270-9.

37 Mitchell AJ, Shiri-Feshki M. Rate of progression of mild cognitive impairment to dementia - meta-analysis of 41 robust inception cohort studies. Acta Psychiatr Scand 2009:119:252-65.

38 Geslani DM, Tierney MC, Herrmann N, et al. Mild cognitive impairment: an operational definition and its conversion rate to Alzheimer's disease. Dement Geriatr Cogn Disord 2005;19:383-9.

39 Jack CR Jr, Knopman DS, Jagust WJ, et al. Hypothetical model of dynamic biomarkers of the Alzheimer's pathological cascade. Lancet Neurol 2010;9:119-28.

40 Hämäläinen $\mathrm{A}$, Tervo $\mathrm{S}$, Grau-Olivares $\mathrm{M}$, et al. Voxel-based morphometry to detect brain atrophy in progressive mild cognitive impairment. Neuroimage 2007;37:1122-31.

41 Karas GB, Scheltens P, Rombouts SARB, et al. Global and local gray matter loss in mild cognitive impairment and Alzheimer's disease. Neuroimage 2004;23:708-16.

42 Jack CR Jr, Petersen RC, Xu Y, et al. Rates of hippocampal atrophy correlate with change in clinical status in aging and AD. Neurology 2000;55:484-9.

43 Jack CR Jr, Weigand SD, Shiung MM, et al. Atrophy rates accelerate in amnestic mild cognitive impairment. Neurology 2008;70(Pt 2):1740-52.

44 Mosconi L. Brain glucose metabolism in the early and specific diagnosis of Alzheimer's disease. FDG-PET studies in MCl and AD. Eur I Nucl Med Mol Imaging 2005;32:486-510.

45 Jack CR Jr, Lowe VJ, Weigand SD, et al. Alzheimer's Disease Neuroimaging Initiative. Serial PIB and MRI in normal, mild cognitive impairment and Alzheimer's disease: implications for sequence of pathological events in Alzheimer's disease. Brain 2009;132(Pt 5):1355-65.

46 Forsberg A, Engler H, Almkvist O, et al. PET imaging of amyloid deposition in patients with mild cognitive impairment. Neurobiol Aging 2008;29:1456-65.

47 Pike KE, Savage G, Villemagne VL, et al. Beta-amyloid imaging and memory in non-demented individuals: evidence for preclinical Alzheimer's disease. Brain 2007:130(Pt 11):2837-44.

48 Fjell AM, Westlye LT, Amlien I, et al. High-expanding cortical regions in human development and evolution are related to higher intellectual abilities. Cereb Cortex 2015;25:26-34

49 Morris JC, Roe CM, Xiong C, et al. APOE predicts amyloid-beta but not tau Alzheimer pathology in cognitively normal aging. Ann Neurol 2010;67:122-31.

50 Dickerson BC, Sperling RA. Neuroimaging biomarkers for clinical trials of disease-modifying therapies in Alzheimer's disease. NeuroRx 2005;2:348-60.

51 Matthews FE, Brayne C, Lowe J, et al. Epidemiological pathology of dementia: attributable-risks at death in the Medical Research Council Cognitive Function and Ageing Study. PLOS Med 2009;6:e1000180.

52 Schneider JA, Arvanitakis Z, Bang W, et al. Mixed brain pathologies account for most dementia cases in community-dwelling older persons. Neurology 2007:69:2197-204.

53 Román GC. Facts, myths, and controversies in vascular dementia. J Neurol Sci 2004;226:49-52.

54 Gorelick PB, Scuteri A, Black SE, et al. Vascular contributions to cognitive impairment and dementia: a statement for healthcare professionals from the American Heart Association/American Stroke Association. Stroke 2011:42:2672-713.

55 Sachdev P, Kalaria R, O'Brien J, et al. Diagnostic criteria for vascular cognitive disorders: a VASCOG statement. Alzheimer Dis Assoc Disord 2014;28:206-18.

56 Wardlaw J, Smith C, Dichgans M. Microbleeds in cerebral small vessel diseaseauthors' reply. Lancet Neurol 2013;12:736-7. 
57 Du AT, Schuff N, Laakso MP, et al. Effects of subcortical ischemic vascular dementia and $A D$ on entorhinal cortex and hippocampus. Neurology 2002;58:1635-41.

58 de Laat KF, Tuladhar AM, van Norden AG, et al. Loss of White matter integrity is associated with gait disorders in cerebral small vessel disease. Brain 2011;134(Pt 1):73-83.

59 Inzitari D, Pracucci G, Poggesi A, et al. Changes in White matter as determinant of global functional decline in older independent outpatients: three year follow-up of LADIS (leukoaraiosis and disability) study cohort. BMJ 2009;339:b2477.

60 Debette $S$, and Markus HS. The clinical importance of white matter hyperintensities on brain magnetic resonance imaging: systematic review and meta-analysis. BMJ 2010:341:c3666.

61 Frisoni GB, Galluzzi S, Pantoni L, et al. The effect of white matter lesions on cognition in the elderly — small but detectable. Nat Clin Pract Neurol 2007;3:620-7.

62 Lee AY. Vascular dementia. Chonnam Med J 2011;47:66-71.

63 Koga H, Takashima Y, Murakawa R, et al. Cognitive consequences of multiple lacunes and leukoaraiosis as vascular cognitive impairment in community-dwelling elderly individuals. J Stroke Cerebrovasc Dis 2009;18:32-7.

64 Benisty S, Gouw AA, Porcher R, et al. Location of lacunar infarcts correlates with cognition in a sample of non-disabled subjects with age-related White-matter changes: the LADIS study. J Neurol Neurosurg Psychiatry 2009;80:478-83.

65 Vermeer SE, Longstreth WT Jr, Koudstaal PJ. Silent brain infarcts: a systematic review. Lancet Neurol 2007;6:611-19.

66 Werring DJ, Frazer DW, Coward LJ, et al. Cognitive dysfunction in patients with cerebral microbleeds on T2*-weighted gradient-echo MRI. Brain 2004;127(Pt 10):2265-75.

67 Brundel M, Kwa VI, Bouvy WH, et al. Cerebral microbleeds are not associated with long-term cognitive outcome in patients with transient ischemic attack or minor stroke. Cerebrovasc Dis 2014;37:195-202.

68 Eckerström C, Olsson E, Klasson N, et al. High White matter lesion load is associated with hippocampal atrophy in mild cognitive impairment. Dement Geriatr Cogn Disord 2011;31:132-8.

69 Allen N, Berry JD, Ning H, et al. Impact of blood pressure and blood pressure change during middle age on the remaining lifetime risk for cardiovascular disease: the cardiovascular lifetime risk pooling project. Circulation 2012;125:37-44.

70 Patel B, Markus HS. Magnetic resonance imaging in cerebral small vessel disease and its use as a surrogate disease marker. Int I Stroke 2011:6:47-59.

71 Brickman AM, Provenzano FA, Muraskin J, et al. Regional white matter hyperintensity volume, not hippocampal atrophy, predicts incident Alzheimer disease in the community. Arch Neurol 2012;69:1621-7.

72 Nitkunan A, Barrick TR, Charlton RA, et al. Multimodal MRI in cerebral small vessel disease: its relationship with cognition and sensitivity to change over time. Stroke 2008;39:1999-2005.

73 Grafton ST, Sumi SM, Stimac GK, et al. Comparison of postmortem magnetic resonance imaging and neuropathologic findings in the cerebral white matter. Arch Neurol 1991:48:293-8.

74 Korczyn AD, Vakhapova V, Grinberg LT. Vascular dementia. J Neurol Sci 2012;322:2-10.

75 Beeri MS, Ravona-Springer R, Silverman JM, et al. The effects of cardiovascular risk factors on cognitive compromise. Dialogues Clin Neurosci 2009;11:201-12.

76 Lockhart SN, Luck SJ, Geng J, et al. White matter hyperintensities among older adults are associated with futile increase in frontal activation and functional connectivity during spatial search. PLOS ONE 2015;10:e0122445.

77 Schaefer A, Quinque EM, Kipping JA, et al. Early small vessel disease affects frontoparietal and cerebellar hubs in close correlation with clinical symptoms-a resting-state fMRI study. J Cereb Blood Flow Metab 2014;34:1091-5.

78 Kirshner HS. Frontotemporal dementia and primary progressive aphasia, a review. Neuropsychiatr Dis Treat 2014;10:1045-55.

79 Neary D, Snowden JS, Gustafson L, et al. Frontotemporal lobar degeneration: a consensus on clinical diagnostic criteria. Neurology 1998;51:1546-54.

80 Rosen HJ, Allison SC, Schauer GF, et al. Neuroanatomical correlates of behavioura disorders in dementia. Brain 2005;128(Pt 11):2612-25.

81 Rosen HJ, Gorno-Tempini ML, Goldman WP, et al. Patterns of brain atrophy in frontotemporal dementia and semantic dementia. Neurology 2002;58:198-208.

82 Seeley WW, Crawford RK, Zhou J, et al. Neurodegenerative diseases target large-scale human brain networks. Neuron 2009;62:42-52.

83 Gorno-Tempini ML, Dronkers NF, Rankin KP, et al. Cognition and anatomy in three variants of primary progressive aphasia. Ann Neurol 2004;55:335-46.

84 Rohrer JD, Ridgway GR, Crutch SJ, et al. Progressive logopenic/phonological aphasia: erosion of the language network. Neuroimage 2010;49:984-93.
85 McKeith L, Galasko D, Kosaka K, et al. Consensus guidelines for the clinical and pathologic diagnosis of dementia with Lewy bodies (DLB): report of the consortium on DLB international workshop. Neurology 1996;47:1113-24

86 Collerton D, Burn D, McKeith I, et al. Systematic review and meta-analysis show that dementia with Lewy bodies is a visual-perceptual and attentional-executive dementia. Demen Geriatr Cogn Disord 2003;16:229-37.

87 Sabattoli F, Boccardi M, Galluzzi S, et al. Hippocampal shape differences in dementia with Lewy bodies. Neuroimage 2008:41:699.

88 Kantarci K, Ferman TJ, Boeve BF, et al. Focal atrophy on MRI and neuropathologic classification of dementia with Lewy bodies. Neurology 2012;79:553-6010.

89 Bozzali M, Falini A, Cercignani M, et al. Brain tissue damage in dementia with Lewy bodies: an in vivo diffusion tensor MRI study. Brain 2005;128(Pt 7):1595-604.

90 Mak E, Su L, Williams GB, et al. Neuroimaging characteristics of dementia with Lewy bodies. Alzheimers Res Ther 2014;6:18.

91 Albin RL, Minoshima S, D'Amato CJ, et al. Fluoro-deoxyglucose positron emission tomography in diffuse Lewy body disease. Neurology 1996;47:462-610.

92 Gomperts SN, Rentz DM, Moran E, et al. Imaging amyloid deposition in Lewy body diseases. Neurology 2008;71:903-10.

93 Donaghy P, Thomas AJ, O'Brien JT. Amyloid PET imaging in Lewy body disorders. Am J Geriatr Psychiatry 2015;23:23-37.

94 Kenny ER, Blamire AM, Firbank MJ, et al. Functional connectivity in cortical regions in dementia with Lewy bodies and Alzheimer's disease. Brain 2012;135:569-81.

95 Sorbi S, Hort J, Erkinjuntti T, et al. EFNS-ENS guidelines on the diagnosis and management of disorders associated with dementia. Eur J Neurol 2012;19:1159-79.

96 McKeith IG, Dickson DW, Lowe J, et al. Diagnosis and management of dementia with Lewy bodies: third report of the DLB Consortium. Neurology 2005;65:1863-72.

97 Jack CR, Barkhof F, Bernstein MA, et al. Steps to standardization and validation of hippocampal volumetry as a biomarker in clinical trials and diagnostic criterion for Alzheimer's disease. Alzheimers Dement 2011;7:474-485.e4 e474.

98 Bermingham SL. The appropriate use of neuroimaging in the diagnostic work-up of dementia. Ont Health Technol Assess Ser 2014;14:1-67.

99 Silverman DH, Gambhir SS, Huang HW, et al. Evaluating early dementia with and without assessment of regional cerebral metabolism by PET: a comparison of predicted costs and benefits. J Nucl Med 2002;43:253-66.

100 Wollman DE, Beeri MS, Weinberger M, et al. Tolerance of MRI procedures by the oldest old. Magn Reson Imaging 2004;22:1299-304.

\section{Answers}

1. False. MTL atrophy is a hallmark biomarker for AD, however, atrophy can be investigated mainly with structural MRI, whereas FDG-PET allows to highlight areas with reduced ${ }^{18}$ fluorodeoxyglucose metabolism.

2. True. Structural MRI has shown that patients with $\mathrm{MCl}$ can have similar pathological findings to $A D$, and so atrophy can be found also in regions outside MTL, such as the lateral temporal lobes, the medial parietal lobes and lateral associative parietal areas.

3. False. fMRI is still used mainly in a research setting as it has a high variability among individuals and it is still mainly used to compare group of patients with healthy controls.

4. False. The presence of leukoaraiosis/WMH and microbleeds indicates higher risk of cognitive decline, stroke and death; however, these imaging features are not highly specific for vascular dementia and can be present in the normal ageing population.

5. True. Amyloid deposition can be found even in other types of dementia, like dementia with Lewy bodies, and it correlates with cognitive decline. 